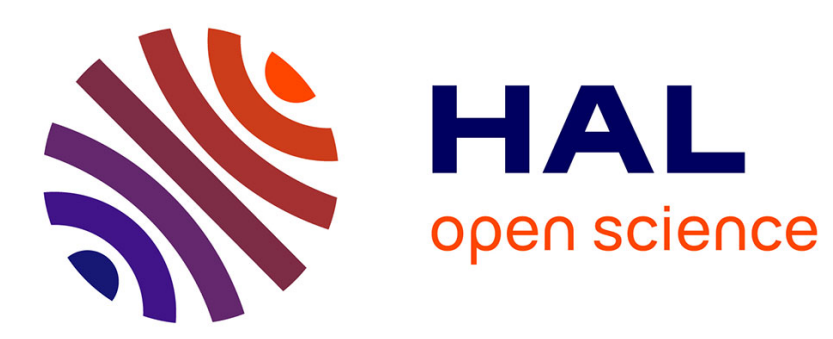

\title{
Packings of deformable spheres
}

\author{
Shomeek Mukhopadhyay, Jorge Peixinho
}

\section{To cite this version:}

Shomeek Mukhopadhyay, Jorge Peixinho. Packings of deformable spheres. Physical Review E: Statistical, Nonlinear, and Soft Matter Physics, 2011, 84, pp.011302. 10.1103/PhysRevE.84.011302 . hal-02296083

\section{HAL Id: hal-02296083 \\ https://hal.science/hal-02296083}

Submitted on 24 Sep 2019

HAL is a multi-disciplinary open access archive for the deposit and dissemination of scientific research documents, whether they are published or not. The documents may come from teaching and research institutions in France or abroad, or from public or private research centers.
L'archive ouverte pluridisciplinaire HAL, est destinée au dépôt et à la diffusion de documents scientifiques de niveau recherche, publiés ou non, émanant des établissements d'enseignement et de recherche français ou étrangers, des laboratoires publics ou privés. 


\title{
Packings of deformable spheres
}

\author{
Shomeek Mukhopadhyay ${ }^{1,2}$ and Jorge Peixinho ${ }^{2,3, *}$ \\ ${ }^{1}$ Chemistry Department, Columbia University, New York, New York 10027, USA \\ ${ }^{2}$ Benjamin Levich Institute, City College of City University of New York, New York, New York 10031, USA \\ ${ }^{3}$ Chemical Engineering Department, City College of City University of New York, New York, New York 10031, USA
}

(Received 13 January 2011; revised manuscript received 17 June 2011; published 13 July 2011)

\begin{abstract}
We present an experimental study of disordered packings of deformable spheres. Fluorescent hydrogel spheres immersed in water together with a tomography technique enabled the imaging of the three-dimensional arrangement. The mechanical behavior of single spheres subjected to compression is first examined. Then the properties of packings of a randomized collection of deformable spheres in a box with a moving lid are tested. The transition to a state where the packing withstands finite stresses before yielding is observed. Starting from random packed states, the power law dependence of the normal force versus packing fraction or strain at different velocities is quantified. Furthermore, a compression-decompression sequence at low velocities resulted in rearrangements of the spheres. At larger packing fractions, a saturation of the mean coordination number took place, indicating the deformation and faceting of the spheres.
\end{abstract}

DOI: 10.1103/PhysRevE.84.011302

PACS number(s): 45.70.-n, 64.60.Cn

\section{INTRODUCTION}

Granular materials have recently emerged as prototypes of the so called "jammed" state [1-3] of matter. The jammed state for a collection of spherical particles assumes that locally the particles are in mechanical equilibrium. However, global constraints may not be satisfied and small perturbations such as tapping may evolve the system toward a global minimum [4]. For a collection of rigid spheres, such a limit point is generally seen to be around 0.64 packing fraction and is known as the random close packing. The physics of jammed hard spheres is developing and it is the purpose of this article to study experimentally a new system of three-dimensional packings of deformable spheres in this context by measuring quantitatively normal forces and contact number distributions.

In two dimensions, experiments on jamming transition using a collection of photoelastic disks [3] showed power law scalings in pressure and contact number distributions as the density of the system increased. There has been limited experimental studies in three dimensions on the properties and structure of dense granular packings [5-8]. Moreover, all experimental studies on three-dimensional systems have been limited to realizations of hard spheres systems. Threedimensional systems of deformable particles are ubiquitous in everyday life, ranging from piles of fruits to arrangements of biological cells. Therefore, developing a simple imaging system with the capability of measuring particle positions, contact areas, coordination numbers, and deformations seems an unique tool to test recent theoretical and numerical results on the jamming of deformable particles $[9,10]$. Although it is possible to perform particle tracking in three dimensions using fluorescent dyes in immersed fluid [7,8,11], severe constraints on optical refractive index matching makes it difficult to resolve particle contacts, and modern investigations resort to $\mathrm{x}$-ray tomography [12] for static studies, magnetic resonance

\footnotetext{
*Present Address: Chimie-ParisTech, and CNRS UMR 7575, 11 rue Pierre et Marie Curie, F-75231 Paris Cedex 05, France.
}

imaging in dense hard-spheres systems [13], and confocal microscopy in colloidal suspensions.

Here we report an analysis of a new three-dimensional imaging technique of disordered packings of deformable particles under normal forces. To achieve this, we used fluorescent particles immersed in an index matched fluid and studied quantitatively the contact number distribution.

\section{EXPERIMENTAL METHODS}

The details of both the normal force and particle contacts as a function of volume fraction were studied using fluorescent polyacrylamide hydrogel spheres. Packings of about 600 deformable spheres of $16 \pm 2 \mathrm{~mm}$ in diameter were considered. The polyacrylamide spheres were first grown in waterRhodamine $6 \mathrm{G}$ solution $(0.05 \mathrm{mg} / \mathrm{ml})$. The polyacrylamide polymer comprised $10 \%$ of the final swelled sphere, so the hydrogel spheres were fluorescent and their refractive index was equal to that of water. Then the spheres were immersed in deionized water within an acrylic square box. The walls were flat and $127 \mathrm{~mm}$ on each side. The box was placed on the normal force transducer of a TA Instrument AR2000 rheometer. The lid of the box was connected to the head of the rheometer, which moved vertically at controlled speeds ranging from 0.1 to $1000 \mu \mathrm{m} / \mathrm{s}$, while the normal force was measured simultaneously. There was a thin gap between the lid and the walls of the box, so that water could escape without disturbing the spheres. The accuracy of the normal force transducer was $0.01 \mathrm{~N}$. For the low packing fractions $\sim 0.60-0.64$, no force transmission was detected by the transducer, although the lid of box was observed to touch one or several spheres. In addition, a vertical green laser sheet was translated through the box in order to image slices through the packing. Photographs of 300 slices ( 3000 by 2000 pixels) through the packing were taken using a digital camera. (The imaging of the arrangement for $\phi=0.64$ is available in the Supplemental Material [14].) Mean coordination numbers and centers of spheres were extracted from the photographs using an image analysis software. 

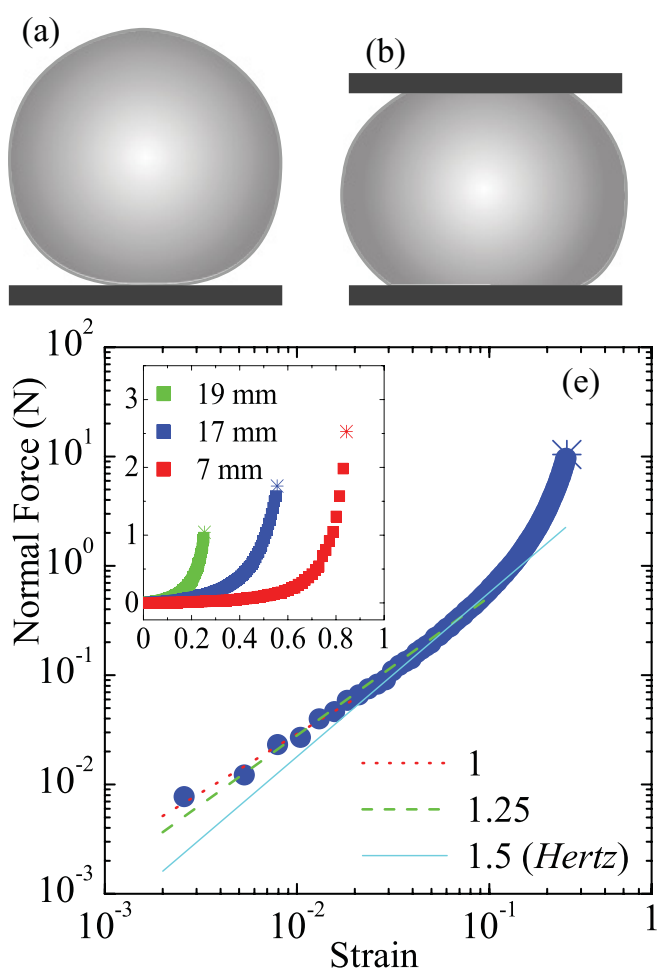

(c)
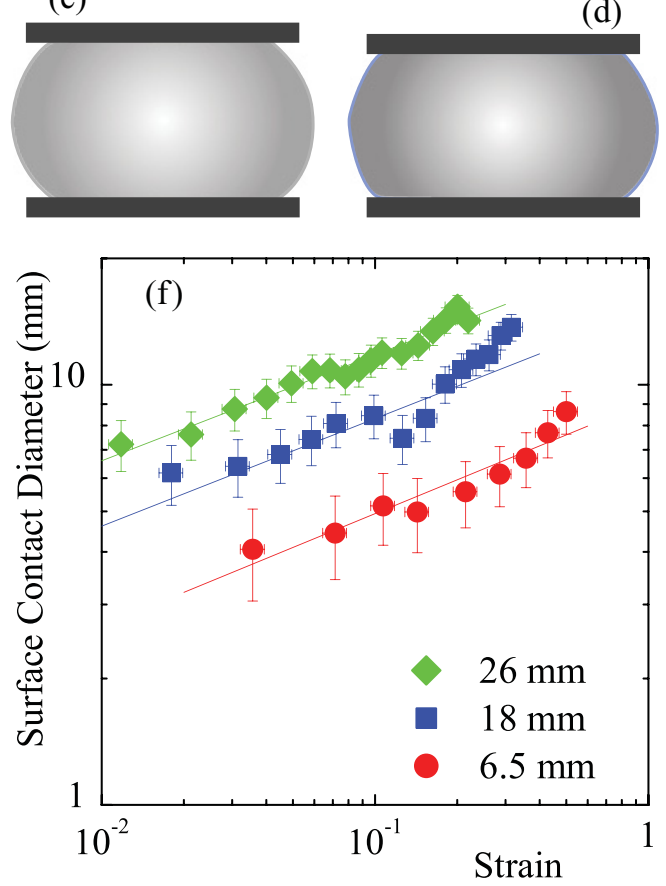

FIG. 1. (Color online) (a)-(d) A sequence of schematics of a 16-mm-diameter sphere compressed at strains of $\varepsilon=0,0.2,0.35$, and 0.4 . (e) Normal force, $F_{N}$, versus strain, $\varepsilon$, for a sphere of $17 \mathrm{~mm}$ diameter. The lines represent fits of $F_{N} \propto \varepsilon^{\alpha}$, where $\alpha$ is the exponent indicated in the legend. Inset: the normal force versus strain with a linear scale for three spheres of different diameters. The stars indicate the fracture of the sphere. (f) Surface contact diameter, $a$, as a function of strain, $\varepsilon$, for three spheres of different diameters. The lines also represent power law fits with exponents of $1 / 4$.

At the scale of a single sphere, normal compression tests were performed using the rheometer and the results are shown on Fig. 1. The individual hydrogel spheres, which can range in diameter from 5 to $30 \mathrm{~mm}$, are highly deformable [see Figs. 1(a)-1(e)]. Elastic spheres are usually dealt with using the approximation of linear equations of continuum elastic theory (Hooke's law) and small contact radius. The Young's Modulus of the hydrogel spheres is $O\left(10^{4}\right) \mathrm{Pa}$ in agreement with previous studies [15]. The Hertz model predicts that the normal force and the strain of the sphere scale as $F_{N} \propto \varepsilon^{3 / 2}$; here $\varepsilon=\left(d_{0}-d\right) / d_{0}$, where $d_{0}$ is the sphere diameter and $d$ is the imposed height. Additionally, the contact region diameter, $a$, scales as $\varepsilon^{1 / 4}$. The properties of the deformable spheres were tested for a range of sphere diameters using different types of surfaces, under dry and immersed conditions, and at different compression velocities. The results are compared to models in Fig. 1(e). For $\varepsilon$ from 0.002 to $0.02, F_{N}$ is proportional to $\varepsilon$. In a larger range of strains, from 0.002 to $0.2, F_{N} \propto \varepsilon^{1.25}$. At large $\varepsilon$, such scaling deviates because of nonlinearities and extensions to the Hertz model need to be used in describing the materials [16]. The surface contact measurement, presented in Fig. 1(f), were obtained by shining a horizontal laser sheet on the horizontal plane between the top of the compressed sphere and the quartz plate. Digital photographs were taken through the plate and analyzed. The contact surface diameter evolved as $a \propto \varepsilon^{1 / 4}$.

In the present investigation, the packings was prepared as follows: the spheres were counted, measured, and gently poured into the water-filled box in order to avoid any trapped air bubble. Before each experimental run, the mixture was stirred with a rod for few seconds. After the decay of any motion, the top lid moved downward for the compression test. The packing fraction was controlled by the position of the lid with a maximum error of 0.02 . Alternatively, an estimate of the packing strain is given by: $\varepsilon_{p} \simeq 1.18 \phi-0.75$. The polydispersity of the system prevented any large-scale crystallization in the system except near the walls of the box. At compression velocities of $100 \mu \mathrm{m} / \mathrm{s}$ or higher, large-scale rearrangements were seen as the packing fraction of the system increased. These correlated motions were reminiscent of slip-plane motion seen in granular materials [17]. However, at lower velocities, such large-scale rearrangements were not observed. The immersed hydrogel spheres form a system that has a small effective coefficient of friction. Measurements of the coefficient of friction for hydrogels on polystyrene surfaces gave values of $O\left(10^{-4}\right)$ [18].

\section{RESULTS AND DISCUSSION}

The normal force was monitored as the packing fraction changed. Examples of regions from photographs halfway inside the packing are shown in Figs. 2(a)-2(c). For $\phi=0.82$, significant deformation and large contact regions between spheres were observed. A three-dimensional reconstruction of a packing at $\phi=0.62$ is also presented in Fig. 2(d). In Fig. 3, typical results for the normal force versus packing fraction 

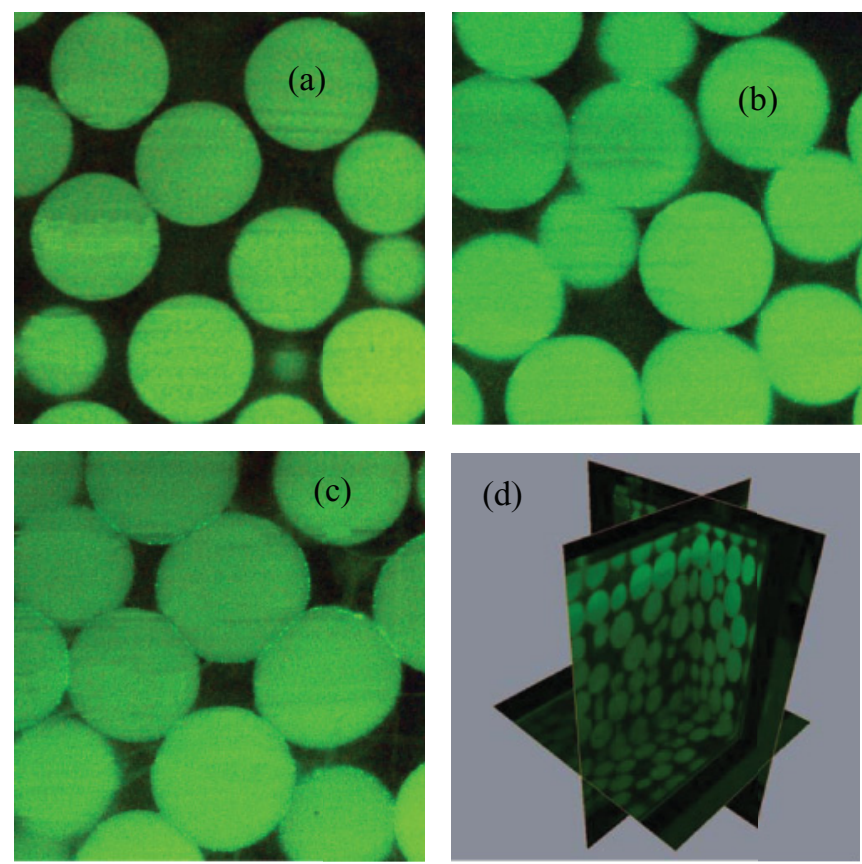

FIG. 2. (Color online) (a), (b), and (c) Region of interest of photographs of compressed packings of deformable spheres $\phi=$ $0.62,0.72$, and 0.82 . (d) Three-dimensional reconstruction of a packing $\phi=0.62$.

are shown for four different compression velocities, which were below the velocities at which large-scale rearrangements occurred. The lines behind the symbols represent power law fits: $F_{N} \propto\left(\phi-\phi_{c}\right)^{\beta}$. The exponent $\beta$ decreases slightly with compression velocity. The inset of Fig. 3 presents the evolution of $\beta$ for a range of compression velocities and the error bars describe the scatter of five to ten runs of the experiments. The scaling exponent, $\beta$, is larger than that observed in two dimensions [3]. The properties of our system are consistent with smaller packings used previously in swollen hydrogel systems [19]. Moreover, the exponent is in between that of

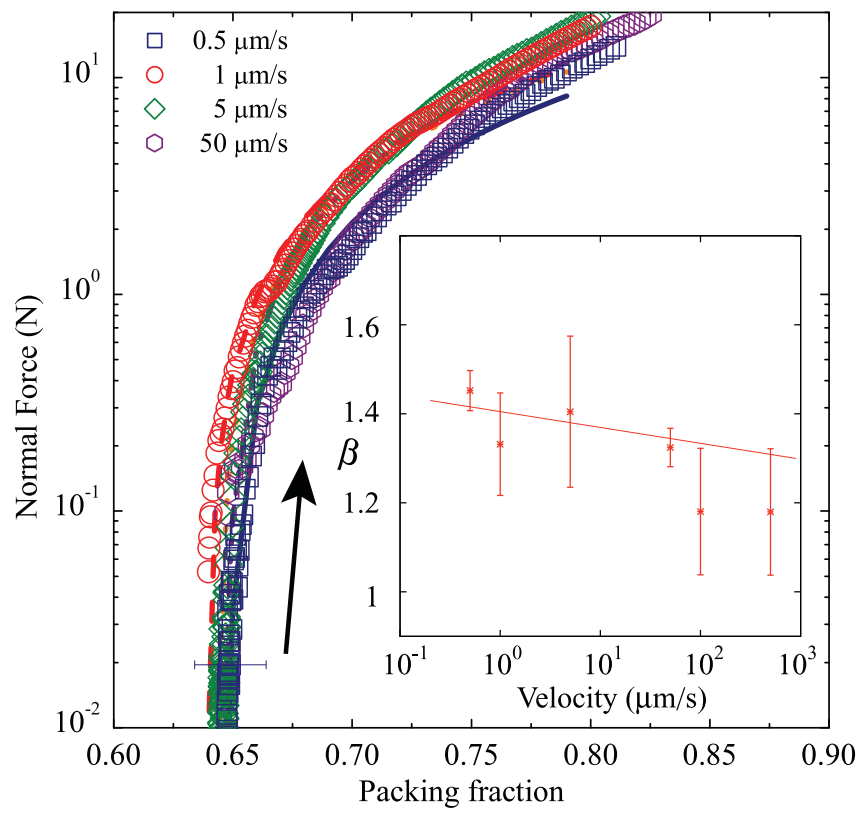

FIG. 3. (Color online) Compression of a packing of deformable spheres. Evolution of the normal force, $F_{N}$, versus the volume fraction, $\phi$. Tests for different compression velocities. The lines represent fits of $F_{N} \propto\left(\phi-\phi_{c}\right)^{\beta}$. Inset: $\beta$ versus compression velocity.

hard sphere systems in three dimensions, which have finite friction $\beta \simeq 1.5[20,21]$ and that of emulsions $\beta \simeq 1.25$, which are usually modeled as frictionless spheres [22]. Since our spheres were Hertzian at relatively low strains, the power law exponent of $\sim 1.4$ is expected if the system responds affinely to compression [9]. The normal force of the packing is determined by the deformability of the spheres rather than the mechanical contacts.

The scanning arrangement allowed the three-dimensional imaging of the packing; the analysis consisted of contact or coordination number as a function of packing fraction and is shown in Fig. 4(a). The error bars represent the measurement
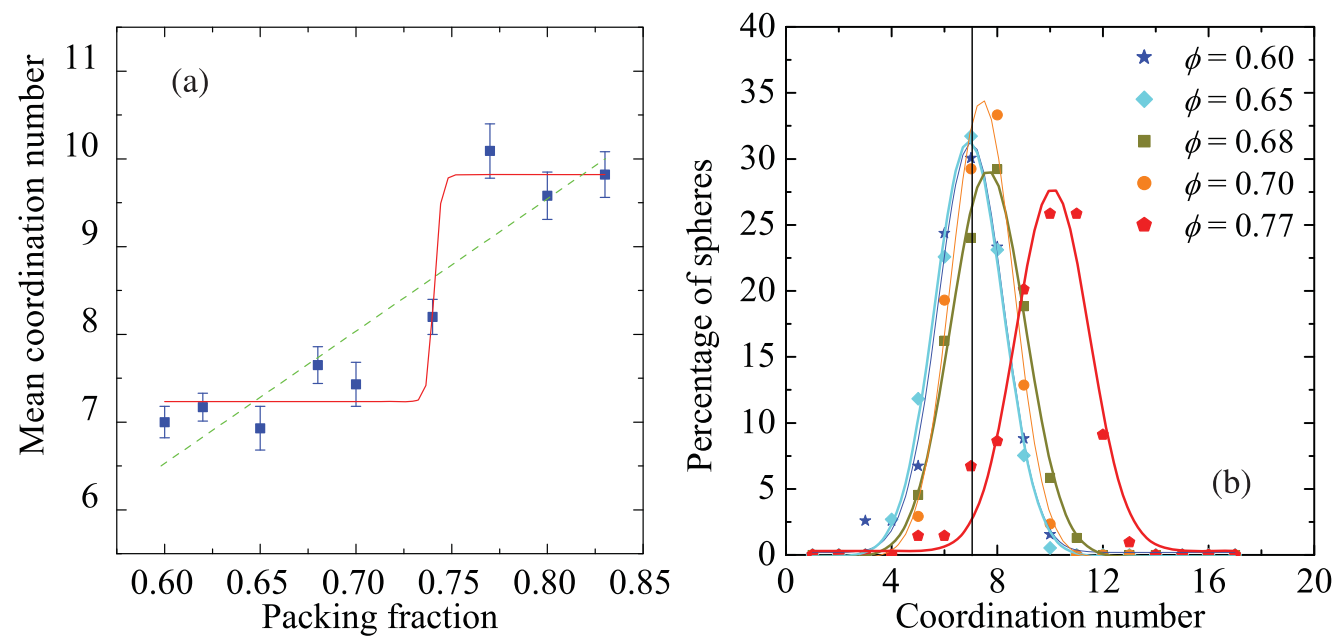

FIG. 4. (Color online) (a) Mean coordination number versus the packing fraction after compression. The dashed line represents the best linear fit. The continuous line represents a sigmoidal fit. (b) Probability distribution of the coordination number for packing fractions from $\phi=0.60$ to 0.77 . The vertical line represents a coordination number of seven. 

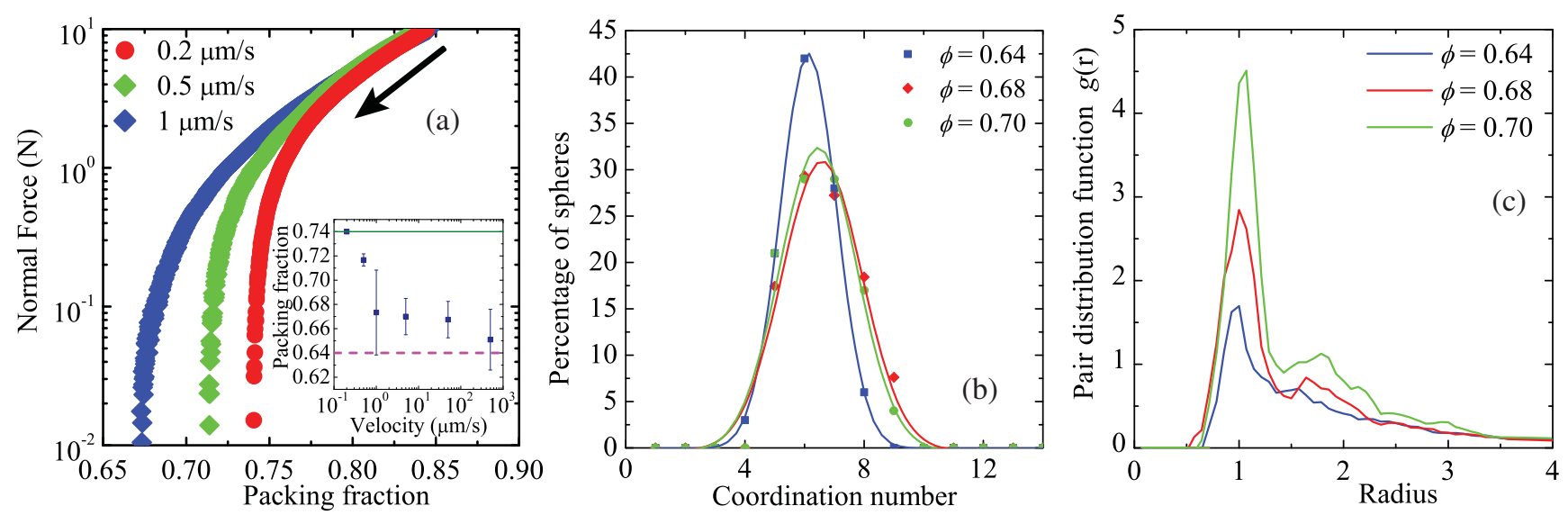

FIG. 5. (Color online) (a) Normal force in packings of deformable spheres at low velocities. Evolution of the normal, $F_{N}$, force versus the volume fraction, $\phi$. Inset: the final packing fraction as a function of the compression-decompression velocity. (b) Probability distribution of the number of contacts for different packing fractions after decompression. (c) Pair distribution function $g(r)$.

error in coordination number. There is contact if the brightness remains constant as one moves radially from one sphere to the adjoining one. The analysis was carried out in the center of the packing without taking into account the three first layers near the walls. The influence of the boundary of the container has been studied by Lachhab and Weill [19] where they tested (i) the height of the packing and (ii) the effect of wall roughness by placing rigid spheres glued on the bottom of the box. They found that the fluctuations upon $\beta$ are $4-7 \%$, which is similar to the errors reported in the present experiments. At the lowest packing fraction $\sim 0.60$ that could be prepared the mean coordination number is slightly above seven and there is a slight increase as the packing fraction increases. The evolution of the coordination number seems linear although a significant jump in coordination number is seen around $\phi=0.74$ and above $\phi=0.80$, where it saturates to almost ten. The jump scenario is supported by a significant faceting of the spheres at packing fractions of 0.77 and higher. Local ordered structures are observed above $\phi=0.74$, but there was no system spanning ordering of the spheres.

The distribution of coordination number can be well described by Gaussian fits as indicated in Fig. 4(b). There is a slight increase of the most probable coordination number as $\phi$ increases. The Gaussian distributions of the coordination number is consistent with numerical simulations of Hertz sphere systems [20,23] for $\phi$ below 0.74 . Then the evolution of the mean coordination number can be described with a sigmoidal evolution [the continuous line in Fig. 4(a)]. This is a strong evidence that deformable spheres packings can differ significantly from the hard sphere results. Such type of behavior was observed in soft particle molecular dynamics simulations independently of the particle number [24]. It suggests that the deformation of the spheres causes the coordination number to saturate at high packing fractions. In the later stage of compression, sphere faceting is important to understand the force network through the packing.

\section{COMPRESSION-DECOMPRESSION EXPERIMENTS}

All our experimental results on the compression of a disordered packings of deformable spheres led to measurable force transmission through the packing from around 0.64 packing fraction independently of the compression velocity. Until now, all the results were restricted to compression of random packings. Now, random packings experienced a compression followed by a decompression for a range of velocities. Surprisingly, the final packing fraction, after decompression from 0.85 packing fraction, reached 0.74 for the lowest velocity $(0.2 \mu \mathrm{m} / \mathrm{s})$. Interestingly, slow decompression led to denser final packing as shown in Fig. 5(a). This is consistent with a recent numerical study of the jamming transition of frictionless spheres [25].

Starting with a packing around 0.64 and performing a compression-decompression test at low velocity, the final mean packing fraction decreased with the decompression velocity as indicated in the inset of Fig. 5(a). It appears that the slower the velocity was, the larger the final packing fraction. Similar packing rearrangements at low velocities after compressiondecompression tests have been observed in other systems with ceramic material in a very viscous resin under cyclic heavy loading [26]. There are two velocity scales in the system: the settling velocity and the elastic velocity. The settling velocity of a sphere sets an upper velocity scale: $V_{s} \sim\left(\rho_{s}-\rho_{f}\right) g d_{0}^{2} / \mu$, where $g$ is the gravity, $\rho_{s}$ is the sphere density, $\rho_{f}$ is the fluid density, and $\mu$ is the dynamic viscosity of water. The settling velocity was of the order of $10^{4} \mu \mathrm{m} / \mathrm{s}$ and corresponds to the velocity to form a loose packing of spheres under gravity. High-velocity compression-decompression tests experienced an asymptotic packing fraction of 0.64 . The other velocity scale is based on the elasticity of the spheres. It is defined by $V_{e}^{2} \sim G / \rho_{s}$, where $G$ is the elastic modulus of the system in pascals. $V_{e}$ was of the order of $10^{-5} \mu \mathrm{m} / \mathrm{s}$, so it is much smaller that any test experimentally achievable. However, at the smallest possible velocities the experimental packing fraction reached the value of $\phi=0.74$, which indicates that slow velocities are necessary to reach a minimum energy state.

A natural question is whether a mean coordination number of six is observed in the packing of deformable spheres. Starting with a random packing and performing a compressiondecompression test at low velocity, the final mean coordination number was six with a narrow width. The distribution remained Gaussian as presented in Fig. 5(b). The structures were more 
ordered, but given the polydispersity, no complete crystallization of the system was observed. This strongly suggests that the mean coordination number is not a unique function of the packing fraction. Therefore, using the mean coordination number as a potential state variable in constructing theories should be treated with caution.

An indication of ordering of the deformable packing after a slow compression-decompression is given by the evolution of the pair distribution function $g(r)$, which describes how the sphere centers are distributed radially at the end of the experiments at slow velocity. In Fig. 5(c), the pair distribution function, $g(r)$, extracted from our image analysis has a single prominent peak. The peak with larger final packing fraction corresponds to the highest peak. Also, the second peak is higher for larger packing fraction in agreement with previous studies [20,27].

\section{CONCLUSIONS}

We have presented an experimental study of amorphous packings of deformable spheres. The mechanical behavior of deformable hydrogel spheres is quantified. At relatively high compression velocities, the system conserves many features of hard sphere packing. A typical power law behavior of the normal force evolution versus packing fraction is found in agreement with previous studies. However, the contact number has a different behavior than the hard sphere system. The novelty of the present system is that a three-dimensional imaging is possible: the mean coordination number and the pair distribution function evolution are quantified. Our system also opens a new way to look at internal dynamics of packings in which the interaction potential and packing fraction can be tuned separately by changing the "stiffness" of the hydrogel. At large packing fractions, the mean coordination number saturates, indicating the deformation and faceting of the spheres.

\section{ACKNOWLEDGMENTS}

The authors are grateful to Bulbul Chakraborty, Mark Shattuck, Jeff Morris, and Iker Zuriguel for discussions. S.M. thanks Matthias Sperl for hospitality at DLR, Cologne.
[1] C. S. O’Hern, L. E. Silbert, A. J. Liu, and S. R. Nagel, Phys. Rev. E 68, 011306 (2003).

[2] A. J. Liu and S. R. Nagel, Nature (London) 396, 21 (1998).

[3] T. S. Majmudar, M. Sperl, S. Luding, and R. P. Behringer, Phys. Rev. Lett. 98, 058001 (2007).

[4] B. Chakraborty and R. P. Behringer, Encyclopedia of Complexity and System Science, edited by Robert A. Meyers (SpringerVerlag, New York, 2009).

[5] C. Song, P. Wang, and H. A. Makse, Proc. Natl. Acad. Sci. USA 102, 2299 (2005); Nature (London) 453, 629 (2008).

[6] T. Aste, Phys. Rev. Lett. 96, 018002 (2006).

[7] S. Slotterback, M. Toiya, L. Goff, J. F. Douglas, and W. Losert, Phys. Rev. Lett. 101, 258001 (2008).

[8] K. A. Lorincz and P. Schall, Soft Matter 6, 3044 (2010).

[9] C. S. O'Hern, S. A. Langer, A. J. Liu, and S. R. Nagel, Phys. Rev. Lett. 88, 075507 (2002).

[10] M. van Hecke, J. Phys. Condens. Matter 22, 033101 (2010).

[11] A. V. Orpe and A. Kudrolli, Phys. Rev. Lett. 98, 238001 (2007).

[12] M. Tsukahara, S. Mitrovic, V. Gajdosik, G. Margaritondo, L. Pournin, M. Ramaioli, D. Sage, Y. Hwu, M. Unser, and T. M. Liebling, Phys. Rev. E 77, 061306 (2008).

[13] E. Fukushima, Annu. Rev. Fluid Mech. 31, 95 (1999).

[14] See Supplemental Material at http://link.aps.org/supplemental/ 10.1103/PhysRevE.84.011302 for an imaging through the packing.
[15] K. N. Nordstrom, E. Vermeuil, W. G. Ellenbroek, T. C. Lubensky, J. P. Gollub, and D. J. Durian, Phys. Rev. E 82, 041403 (2010).

[16] Y. Tatara, S. Shima, and J. C. Lucero, J. Eng. Mater. Tech.113, 287 (1991); K. K. Liu, D. R. Willimas, and B. J. Briscoe, J. Phys. D 31, 294 (1998); E. C. Muniz and G. Geuskens, Macromolecules 34, 4480 (2001); Y.-L. Lin, D.-M. Wang, W.-M. Lu, Y.-S. Lin, and K.-L. Tung, Chem. Eng. Sci. 63, 195 (2008).

[17] P. Yu and R. P. Behringer, Chaos 15, 041102 (2005).

[18] J. P. Gong, T. Kurokawa, T. Narita, G. Kagata, Y. Osada, G. Nishimura, and M. Kinjo, J. Am. Chem. Soc. 123, 5582 (2001).

[19] T. Lachhab and C. Weill, Eur. Phys. J. B 9, 59 (1999).

[20] H. P. Zhang and H. A. Makse, Phys. Rev. E 72, 011301 (2005).

[21] H. A. Makse, D. L. Johnson, and L. M. Schwartz, Phys. Rev. Lett. 84, 4160 (2000).

[22] M.-D. Lacasse, G. S. Grest, and D. Levine, Phys. Rev. E 54, 5436 (1996).

[23] Y. Jin and H. A. Makse, Physica A 389, 5362 (2010).

[24] R. Arevalo, I. Zuriguel, and D. Maza, Int. J. Bif. Chaos 19, 695 (2009); Phys. Rev. E 81, 041302 (2010).

[25] P. Chaudhuri, L. Berthier, and S. Sastry, Phys. Rev. Lett. 104, 165701 (2010).

[26] X. Ye, T. Ganley, J. F. Morris, N. Tonmukayakul, and M. A. Parker, J. Petrol. Sci. Eng. 65, 193 (2009).

[27] A. Donev, S. Torquato, and F. H. Stillinger, Phys. Rev. E 71, 011105 (2005). 